\title{
Importance of the Relation of Solid Surfaces and Liquid Films in Some Types of Engineering Construction*
}

\author{
By Clifford Richardson
}

THE importance of surface phenomena as they relate to the stability of earth fills, embankments in relation to water, and more especially in the construction of asphalt pavements and highway surfaces, has only been adequately recognized during the past few years, although attention was called to some features connected therewith as long ago as 1905 by the writer, in "The Modern Asphalt Pavement." It was there demonstrated that the finer the mineral aggregate in a sheet asphalt pavement the greater the surface area of the particles which is to be coated with bitumen and the more satisfactory the pavement.

The great advance that has been made in the last ten years in our comprehension of the relation of solid surfaces and films of liquids in contact therewith, and especially of the relation of solids in a colloidal state to liquids with which they may be associated, makes it possible to apply the information which has been developed to an interpretation of the phenomena which are connected with the rational construction of bituminous road surfaces.

Great energy is displayed by the contact of solid surfaces with liquid films. This is known as surface energy. Where a solid is uniformly dispersed through a liquid this is known as a physical system. In such a system energy is developed and this energy is proportional to the area of surface displayed by the solid and to the character of the liquid in which it is dispersed. The relation of the extent of surface displayed by the solid and the liquid covering it, plays an important part in the surface energy developed in any system consisting of subdivided mineral matter and bitumen in a more or less liquid form. It is of great interest in reaching a rational interpretation of the relations of these materials in the construction of asphalt pavements and roads.

In 1905 it was shown by the writer, in the book which has been mentioned, that the increased extent of surface offered by a fine aggregate as compared with the coarser material, was enormous. One gram of sand, consisting of grains of uniform size which would just pass a 10 mesh sieve and have a diameter of about 2 millimeters would consist of 212 particles having a surface area of 15 square centimeters. If the same weight of material were reduced to a size which would just pass a 200 mesh sieve having openings $.08 \mathrm{~mm}$ in diameter the number of particles would be increased to nearly one and a half million and the surface area would be $283 \mathrm{sq}$. cm. Proceeding still further and reducing the particles to a size of $.002,5 \mathrm{~mm}$. they would number over forty-six billion and the surface area would be more than 9,000 square centimeters. 'The relation of the surface area of the material as a solid mass to that of the surface of the same material when subdivided, is known as specific surface.

As stated by Ostwald, "Surface energy, as usually discussed, is made up of two components: a capacity factor as measured by the absolute surface, and an intensity factor as measured by surface tension. The latter type of surface energy endeavors to decrease the surface of a system if free energy is available."

In a sheet asphalt paving mixture the capacity factor is measured by the degree of fineness of the mineral aggregate, and the intensity factor by that of the character, that is to say, the surface tension, of the bitumen which is in use as a cementing material. In the case of very fine aggregates the increase in the surface area, that is to say, the specific surface, is, as has been demonstrated, enormous, as compared to that of a coarse one and would necessarily call for a larger amount of bitumen to cover it. This fact has an important bearing on asphalt surface mixtures, as prepared from different sands and with different amounts of filler. For example, the mineral aggregate of a coarse New York mixture laid in 1895 was found to have a surface area of about 44 square feet in one pound of material, whereas in a finer one of 1898 this was increased to over 60 square feet. The former was an unsatisfactory, the latter a very satisfactory pavement. At that time attention was called to the fact that the more extended area of the finer aggregate would require a larger percentage of asphalt than the coarser and was desirable on that account.

No further explanations or conclusions as to the nature of the relations involved were then drawn. With the further study, which has been so widely carried on in the last ten years, on the relation of surfaces and films and of the characteristics exhibited by matter in a highly dispersed or colloidal state, we are to-day in a position to comprehend more fully and explain why this extended surface is a matter of importance in the construction of a satisfactory pavement, and why accepted methods of procedure, which have, hitherto, been founded more or less on an empirical basis alone and not upon certain definite physical laws associated therewith, have been successful. Surface phenomena are now recognized as being of great importance. For a thorough comprehension of the subject, reference must be made to the literature which is available. ${ }^{1}$

Investigations in connection with the relation of the surfaces of mineral aggregates of the type employed in the construction of asphalt pavements and of films of bitumen which serve to cement them together and in other engineering problems as well, have proved of interest not only as explaining the reasons for the successful results obtained in the construction of pavements of the type mentioned, as developed empirically during the last twenty years, but also, carrying these principles much further, in developing the possibility of producing other structural materials containing large percentages of mineral matter in a colloidal state, which are of value as presenting unusual physical characteristics and availability for particular uses. It was empirically shown as long ago as 1894 to 1896 by the study of successful surfaces that a satisfactory sheet asphalt pavement was one which contained, as part of the aggregate with which the bitumen is combined, a considerable percentage of filler, that is to say, of extremely fine mineral matter. This was attributed to the large extent of surface which such an aggregate offered to which bitumen might adhere, and in this respect was a correct inference. At that time, however, the relation of surfaces and films and the principles of the chemistry of matter in a colloidal state had not been made clear, while to-day this subject has been more thoroughly elaborated and affords a means for the rational interpretation and explanation of the relation of surfaces and films, as applied to combinations of a mineral aggregate and bitumen, and of the enormous energy developed by the extensive surface presented by solid matter in the colloidal state. This now makes it possible to carry out work of this description on a scientific and rational basis.

One cannot properly speak of a substance, in itself, as a colloid. The word denotes, rather, a state of matter dependent on the relation of one phase to another, and is one in which all material can exist. It relates, as regards solids and liquids, to the state of subdivision of the former and its dispersion through the latter. Whether a solid is in a colloidal state or not depends not only on its state of subdivision but upon the viscosity of the liquid or continuous phase or medium in which it is dispersed. A substance which, owing to its degree of subdivision, would settle rapidly out of water, a mobile aqueous phase, may exist in a colloidal state as regards a very viscous bitumen, such as the heavy residuals of petroleum, which fiow but slowly at ordinary temperatures. An example of the latter will be found in Trinidad asphalt, which contains a large amount of fine mineral matter, 25 per cent., which, all of it, exists in a colloidal state as regards the bitumen, although some of it is fine sand, of a size which will be retained on a screen of 100 meshes to the lineal inch. This comparatively coarse material

1" An Introduction to the Physics and Chemistry of Colloids," by Emil Hatschek, 1916. P. Blakiston's Son \& Co., Philadelphia.
"The Chemistry of Colloids," by W. W. Taylor, 1915. Long-
mands, Green \& Co., New York

"Die Welt der vernachlassigten Dimensionen," by Wolgfang "wald, Theodor Steinkopff, Dresden, Germany.

Hand book of Colloid Chemistry," by Ostwald, translated by cher, 1909. P. Blakiston's Son \& Co., \&Philadelphia.
"The Physical Properties of Colloidal Solutions." "The Physical Properties of Colloidal Solutions,"
Burton, 1916. Longmans, Green \& Co., New York.

Burton, 1916. Longmans, Green \& Co., New York.
"The Technical Control of Colloidal Matter in Clay," Technologic Paper No. 23. Bureau of Standards, Washington, D. C. is in a state known as colloidal, because the continuous phase in which it is dispersed may be regarded as a highly viscous liquid.

Clay, as it occurs in nature, is of a very varied character. It is, in deposits of different origin, in a
more or less colloidal state as regards the water with more or less colloidal state as regards the water with manner in which it has been formed, whether it is residual or sedimentary, the latter being usually the more colloidal.

It is recognized that clay can be readily diffused in water. Dry clay cannot be readily diffused through the denser forms of bitumen because the individual particles of the clay adhere to one another, and because they are covered with an adsorbed film of air or water which prevents its being coated with bitumen. For these reasons dry clay cannot be dispersed uniformly through such a viscous medium as a dense bitumen. If, however, the clay is originally thoroughly dispersed in water as a continuous phase it can then be emulsified with any bitumen which is sufficiently liquid at temperatures below that of boiling water to make it possible. On the removal of the aqueous phase from the emulsion by heating, the highly dispersed clay will then enter into the bituminous phase without difficulty and will then be dispersed therein in a colloidal state or as a colloidal solution of clay in bitumen.

Clay in a colloidal state can also be introduced in the same way with greater facility into the more liquid types of asphaltic bitumen. The amount, introduced in this way, may reach with certain oils as much as 60 per cent. Such a material may be used for fiuxing the harder bitumens for the purpose of introducing colloidal clay into them in a simpler manner than by attempting to do so directly. With a sufficient amount of' clay in a colloidal state in the fiux, the latter ho such stability that it can be shipped without packages. Combinations of asphalt and colloidal clay may be obtained in this way which present some extraordinary and valuable characteristics from an industrial point of riew.

The native asphalts of a highly viscuous nature, and the heavy residuals derived from asphaltic petroleum, possess, however, very different capacities for maintaining clay in suspension in a colloidal state, and this capacity differentiates them in a striking manner, demonstrating the difference in character of the components of which these asphalts are made up. This is illustrated by the determinations of what may be called the capacity of various asphalts to maintain colloidal clay in suspension under different conditions. Colloidal clay was introduced into a number of solid residuals of various types of petroleum, by the addition of enough aqueous clay paste to result in a material which, after removal of the water, should consist of 07 per cent. bitumen and 33 per cent. clay. These materials were maintained in a melted condition, at a temperature of $325^{\circ} \mathrm{F}$. in tubes for 24 hours. The sedimentation which, with the reduction of the viscosity of the continuous phase, went on in that period, appears from the following figures:

\begin{tabular}{|c|c|c|c|c|}
\hline & & & $\begin{array}{l}\text { cent. } \\
\text { Matter }\end{array}$ & $\begin{array}{l}\text { Per cent } \\
\text { Sdei- }\end{array}$ \\
\hline & tra- & Before & After & menta- \\
\hline Trinidad Residual. & & & & \\
\hline Trinidad Residual. & 50 & 33.5 & 33.7 & 0.0 \\
\hline ababui Residual. & 48 & 32.4 & 30.1 & 7.0 \\
\hline Mexican Residual. & 50 & 33.3 & 27.2 & 18.3 \\
\hline California Residual. & 50 & 31.8 & 23.8 & 25.2 \\
\hline $\begin{array}{l}\text { Mid-Continental } \\
\text { Semi-Paraffine }\end{array}$ & & & & \\
\hline & 51 & 33.8 & & 35.8 \\
\hline
\end{tabular}

Colloidal Capacity of Various Types of Bitumen 67 Per Cent. Bitumen and 33 Per Cent. Clay,
Wet, Maintained at 325 F. for 24 Hours

The colloidal capacity thus determined is a function of the character of the particular bitumen and also of its viscosity at the temperature concerned. The strikine feature is that the residual asphalt prepared from semf. paraffine petroleum of the Mid-continental field deposited 35.8 per cent. of the clay at high temperature, showing the small capacity of paraffine oils to maintain clay in suspension in a colloidal state. At the other extreme a Trinidad residual deposits nothing under the same circumstances. A Bababui residual, 
prepared from a Venezuelan petroleum, which is heavier than water when it emerges from the well, deposits but 7 per cent., a Mexican residual 18.3 per cent. while the California residual deposits an in creased amount.

As the film of bitumen which covers the mineral aggregate of a sheet asphalt pavement is formed at the temperature given, these data may convey some idea as to the capacity of the different materials for coating the grains of this aggregate, but too much dependence must not be placed upon them as there are so many other controlling features in relation to the character of the films. It is striking, however, that the Trinidad residual is so thoroughly differentiated from all the others, thus confirming the opinion, based upon service tests in regard to the unique character of this material.

If the same mixtures of bitumen and colloidal clay are examined in a 10 per cent. solution in carbon-disulphide, which has been submitted to centrifugal action the results are equally striking.

Sedimentation of 10 Per Cent. Solutions on Centrif uging In Carbon-Disulphide

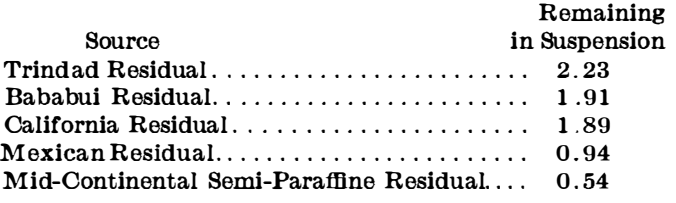

Colloidal Capacity of Various Types of Bitumen

In Mixtures of 67 Per Cent. Bitumen and 33 Per Cent.
Colloidal Clay

The Trinidad residual again has the greater colloidal capacity, while the Mexican residual, under the conditions, shows a decided reduction in this respect, while the semi-paraffine, Mid-continental material, again has the smallest capacity. This capacity of different bitumens of the same consistency for maintaining clay in a colloidal state, under different conditions, is strong evidence of the fact that asphalt from various sources is of a different character.

Trinidad lake asphalt after the removal of the water by heating, that is to say, in a refined condition, contains about 35 per cent. of anhydrous mineral matter, Is determined by ignition, all of which must have been in a colloidal state, since the amount found in all parts of the deposit is the same and this uniformity can only be accounted for on this supposition. If this asphalt is dissolved in carbon-disulphide or benzol to form a solution of varying degrees of concentration and centrifuged to remove the mineral matter which is too coarse to remain in the colloidal state, in a liquid of the viscosity corresponding to the concentration, the resulting solutions will be found to contain a percentage of colloidal matter varying with the concentration and viscosity. The data which have been obtained as the re sults of experiments along these lines are as follows:

\begin{tabular}{|c|c|c|c|c|}
\hline Per cent Concen & $\begin{array}{l}\text { Spec. } \\
\text { Grav. }\end{array}$ & $\begin{array}{l}\text { Increase } \\
\text { for } 1\end{array}$ & $\begin{array}{l}\text { Absolute } \\
\text { Viscosity }\end{array}$ & $\begin{array}{l}\text { bsolute } \\
\text { for } 1\end{array}$ \\
\hline Benzol. ...... & .876 & & 0.00652 & \\
\hline 1 per cent T.R.A. & .877 & .0010 & 0.00654 & 0.00002 \\
\hline 2 per cent T.R.A & .879 & .0020 & 0.00687 & 0.00033 \\
\hline 5 per cent T.R.A & .883 & .0013 & 0.00759 & 0.00024 \\
\hline 10 per cent T.R.A & .889 & .0012 & 0.00961 & 0.00040 \\
\hline 20 per cent T.R.A. & .911 & .0022 & 0.01269 & 0.00067 \\
\hline 30 per cent T.R.A & .930 & .0019 & 0.04198 & $0.0025 ?$ \\
\hline 40 per cent T.R.A & .957 & .0027 & 0.09477 & 0.00521 \\
\hline 50 per cent T.R A & 1.012 & .0055 & 0.31800 & 0.02240 \\
\hline & 1.400 & .0076 & & \\
\hline
\end{tabular}

Characterization of Solutions of Trinidad Asphalt

\begin{tabular}{|c|c|c|}
\hline & $\begin{array}{l}\text { Refined } \\
\text { per cent } \\
\text { Colloidal }\end{array}$ & $\begin{array}{l}\text { Refined } \\
\text { amount } \\
\text { for }\end{array}$ \\
\hline Per cent Concentration & & \\
\hline 1 per cent $\ldots \ldots \ldots$ & 2.54 & 2.54 \\
\hline 1 per cent. & 2.54 & 2.54 \\
\hline 2 per cent. & 2.01 & 1.00 \\
\hline 5 per cent. & 2.09 & .42 \\
\hline 10 per cent. . & 2.73 & .27 \\
\hline 20 per cent.. & 3.13 & .16 \\
\hline 30 per cent. & 4.19 & .14 \\
\hline $40 \mathrm{per}$ & 6.51 & .16 \\
\hline 50 per cent.. & 10.69 & .21 \\
\hline 100 per cent. & 35.40 & .35 \\
\hline
\end{tabular}

\section{Characterization of Solutions of Trinidad Asphalt}

The percentage of clay in a colloidal state varies but slightly for concentration of from 1 to 10 per cent., but fincreases gradually above this. These changes are most 1 per cent. for the different intervals, as shown in the tables. The amount decreases gradually per unit of bitumen until the concentration of 40 per cent. is reached, when it again increases up to 100 per cent.. which is not, however, a maximum.

It appears from these figures that this result might be due to irregularities in changes in the viscosity of the solutions under examination, with concentration, but determinations have shown that this is not the case, since the absolute viscosity increases in a perfectly regular way. The same may be said in regard to the increase of the specific gravity of the solution. On plotting these data it appears that the curves show that the changes in specific gravity are represented by a straight line, or approximately so, the curve for absolute viscosity by a paraboloid, and that for suspended solids by a slightly irregular paraboloid. It appears then that the increase in the amount of clay in a colloidal state is gradual for a lower but sharp for the higher concentrations.

The peculiar relations of colloidal clay and bitumen as demonstrated by the preceding data are of great interest. They serve to demonstrate the importance of the relations of surfaces and films and of the peculiar role played by matter in a colloidal state in connection with combinations of bitumen and mineral matter as employed in paving and road construction. It can be readily seen that the relations of water as a continuous phase and mineral matter in various degrees of dispersion can be interpreted along similar lines and are of importance to the engineer in connection with the stability of earth dams, fills, embankments and other structural relations of materials of this type. It is a subject which, in this direction, has received only superficial attention up to the present time.

\section{The Hippocampus}

The interesting little fish known as the seahorse or Hippocampus, which has attracted much attention on account of its curious form and no less peculiar habits from time immemorial, is discussed by Dr. Charles R. Eastman, of the American Museum of Natural History, in one of the Smithsonian Annual Report papers just issued.

Occurring plentifully in the Mediterranean, the typical species was well known to the ancients; and owing to the wide distribution of the remaining thirty-odd species composing the genus, seahorses have now become familiar objects in most large aquariums of the world. They appear to be a weird combination of a horse's head and a body or tail of a caterpillar. It has been said that if a coiled worm were attached to the base of chess knight the seahorse would be well imitated.

We owe to the late Dr. Theodore N. Gill, Associate in Zoology of the U. S. National Museum, a fine account of the life history of the Hippocampus, published in the proceedings of the U. S. National Museum in 1905 The movements, feeding and breeding habits of these creatures are all extremely curious, and are well described by Dr. Gill.

The most frequent position assumed is a state of rest, with the tail wound around the stem of a plant or other substance, and the body carried nearly or quite erect, but notwithstanding the apparent rigidity of its cuirasslike body armor, almost every other attitude consistent with such a form can be assumed. The body can be thrown outward or downward at various angles, the tail being wound around a plant in a double coil. Once in a while one eye may roll toward the observer, while the other may be passive or look backward or even in the opposite direction. The little fish can move its eyes independently of each other, and in entirely different ways. A comical effect is produced by the manner in which these fishes peer at some objects, reminding one of a very near-sighted person.

Releasing itself from its support it can progress slowly, still in a vertical position, its tail curved inward, its dorsal fin rapidly undulating, reminding one of a screwpropeller, and with its pectoral fins vibrating in harmony.

Incased as it is in an almost inflexible coat of mail, progression can not be effected by lateral flexion of the body as in ordinary fishes, and with the flexion in body as in ordinary fishe
vertical direction is limited.

With such limited powers of progression a nice adjustment of organs is necessary. One method is said to use an air bladder, comparatively large and always distended by a quantity of gas so exactly in harmony with the specific gravity of the body that this entire body is a hydrostatic apparatus of extreme sensibility. A proof of this is that if a single bubble of gas no larger than the head of a very small pin be extracted, the fish immediately loses its equilibrium and sinks to the bottom on which it must crawl until the wound has been cicatrized and a new supply of gas secreted by the internal membrane new supply of gas secreted by the internal membrane
of the bladder. It is well known that the eggs of Hippoof the bladder. It is well known that the eggs of Hippo-
campus are taken care of by the male in an abdominal sac or pouch.

The name Hippocampus is derived from the Greek words hippos, signifying horse, and kampos or kampe, a caterpillar. And, in fact, the head is shaped and the neck arched like those of a horse, while the body or tail resembles a caterpillar.
One sixteenth centur writer states that: Its size does kin, and neither man nor fish of other kind esteem it as food. In color it is sometimes dark; in other cases food. In color it is sometimes dark; in other cases
white. It bears a small fin, a little elevated along the back, and another small one on the neck where it joins the head. The mouth is small and tubiform. Dead or dried specimens have the tail coiled inward, like that of a chemeleon; it is furnished with small, blunt prickles, and is of quadrangular section. The spinuos projections arise from transverse folds which cross the tail

Certain authors profess that the ashes of Hippocampus when commingled with liquid pitch, tallow, or oil of sweet majoram, cure baldness and pain in the sides. An application of oil of roses into which the live animal has been dipped and killed is an efficacious remedy for chills and fever.

Dr. Eastman mentions alleged medicinal properties of Hippocampus, gravely set forth by Matthioli, Belon, Rondelet, Gesner and others. Aristotle does not mention the Hippocampus, and this word was used by the poets of classical antiquity as the name of a sea monster, half horse and half fish, on which sea divinities rode. It is probably in this character that conventionalized representations of the creature appear in Etruscan and other ancient works of art.

A curious confusion of this odd fish with some other species occurred years ago, when its identity was mixed with the Remora, or sucking-fish, also known as "Shipstayer," which clove to ships and held them steadfast in the sea, as though the ships were aground, although the wind and waves strove to wreck them. Another story connected with the Hippocampus with the eel-like "Reversus," said to have a cap attached to its head with which it captured other fish and tortoise, and was therefore used by the natives, reported by Columbus for catching other fish. It also was once called "sea dragon," or "Zydeath," and again, from the Latin, "Equus maris," "Horse of the Sea," on account of the act that these creatures were all supposed to die in the air or at least suffer when out of water.

\section{Passive State of Metals}

THE results obtained by previous investigators, discussed in detail, are claimed to justify the view that passivity in all cases is caused by oxidation, but the existing theories are regarded as insufficient to account for the facts observed. It is submitted that the true cause of passivity is the formation of a higher oxide (compare Armstrong and Colgate, this J., 1913, 394) which is adsorbed by the metal to form a protective layer more noble than the metal itself. Very small amounts of oxide are considered sufficient to bring about passivity, and in most cases probably the oxide formed is stable only in the adsorbed state. The disappearance of passivity at high temperatures is thus accounted for by the decomposition of the oxide which accompanies diminished adsorption, and the slow change of E. M. F. as passivity develops is explained by the fact that the potential of the oxide produced has its maximum effect only when passivity is complete; other facts, such as those observed by Heathcote (this J., 1907, 899) are shown likewise to accord with the theory. When iron is rendered passive in nitric acid, it is supposed that $\mathrm{Fe}^{*}$ ions are produced and oxidized to $\mathrm{Fe}^{\cdots}$ ions, and that then some of the $\mathrm{Fe}^{\cdots}$ ions are further oxidized (probably to give the oxide $\mathrm{FeO}_{2}$ ) and the higher oxide is adsorbed; the effect of vigorous stirring is to circulate the $\mathrm{Fe}^{\cdots}$ ions before further oxidation can take place, oxide in the case of chromium is not higher than the trioxide $\mathrm{CrO}_{3}$, for the metal becomes passive in a solution of chromic acid; it is probably the dioxide $\mathrm{CrO}_{2}$. Lead was rendered passive without the appearance of a visible oxide film, but it is thought that the process is essentially similar whether an obvious coating is proby C. W. Bennet and W. S. Burnham, in Z. Elektrochem.

\section{Hard Rubber Sponge}

HARD rubber sponge is prepared from soft rubber sponge by further vulcanization. The stock is made up with sufficient sulphur to effect the transformation to ebonite, so that after producing soft rubber further ulcanization takes it over to hard sponge.

This material has an apparent specific gavity of 0.2 to 0.065 , which is $1 / 2$ to $3 / 4$ that of cork, and $1 / 4$ to $1-12$ that of wood. It may be worked in any way customary with hard rubber, such as sawing, boring, machining, etc. It still has the cellular structure of soft sponge, and therefore its insulating properties are not in the east affected. Hard rubber sponge is suggested as an insulator for covering flasks, or ice box-walls. As it has considerable strength, it is proposed as a material for aeroplane framework and for automobile bodies. 